\title{
Capability of FDEM for Journal Bearings with Microstructured Surface
}

\author{
Numerical Simulation of Journal Bearings with Turbulence and Inertia Effects \\ Torsten Adolph and Willi Schönauer
}

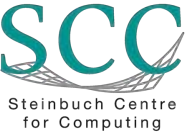

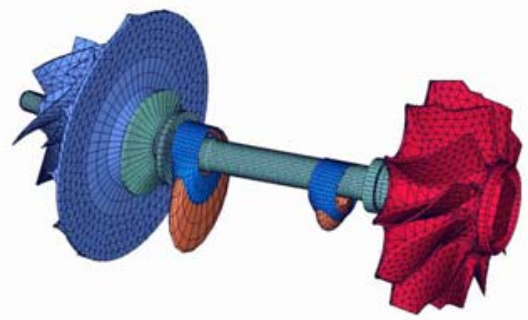

Source: „Mehrkörperdynamische Modellierung von Full Floating Schwimmbuchsengleitlagern", GTT-Fachtagung 2007
Thermal turbo-engines, e.g. turbo-superchargers, have to be driven by high rotational speeds to realize good combustion efficiencies. These rotors are often pivoted by journal bearings, particularly when they are running with high rotational speeds. Above a specific rotational speed, the rotors get instable. This critical speed depends on different bearing and system parameters and can only be determined for simplified models. Current software solutions to simulate journal bearings are based on the so-called simplified Reynolds differential equation, which follows from the equation of momentum and the continuity equation under conditions of Stokes flows.

FDEM - Finite Difference Element Method: Software for the numerical solution of partial differential equations FDEM is a black-box solver for nonlinear systems of elliptic and parabolic partial differential equations in 2D and 3D that generalizes the Finite Difference Method on arbitrary geometry and that contains an estimate of the error of the solution. In many technical applications, the solution domain consists of several subdomains. In FDEM, these subdomains are separated by dividing lines which are internal boundaries where the subdomains are connected by coupling conditions. FDEM is efficiently parallelized on distributed memory parallel computers by MPI and thus, the code is portable to an extremely high degree and runs under Unix/Linux and Windows. We want to solve the

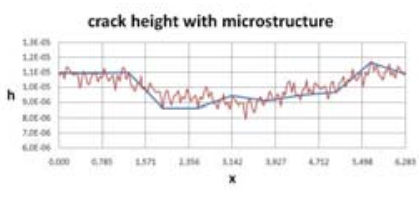

p [Pa]

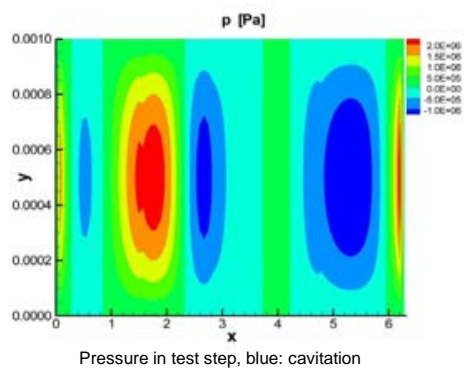

Reynolds equation with nonlinear inertia terms

$\frac{\partial}{\partial x}\left(G_{x} \frac{\rho h^{3}}{\eta} \frac{\partial p}{\partial x}\right)+\frac{\partial}{\partial y}\left(G_{y} \frac{\rho h^{3}}{\eta} \frac{\partial p}{\partial y}\right)-\frac{\partial}{\partial x}\left(G_{x} \rho h u_{m}\right)-\frac{\partial}{\partial y}\left(G_{y} \rho h v_{m}\right)-\rho w=$ $-\frac{\partial}{\partial x}\left(G_{x} \frac{\rho h^{2}}{\eta}\left(V_{x} w+\frac{\partial I_{x x}}{\partial x}+\frac{\partial I_{x y}}{\partial y}\right)\right)-\frac{\partial}{\partial y}\left(G_{y} \frac{\rho h^{2}}{\eta}\left(V_{y} w+\frac{\partial I_{y x}}{\partial x}+\frac{\partial I_{y y}}{\partial y}\right)\right)$

Turbulence factors: Bulk velocities: Momentum fluxes: Flow velocities (given): Crack height:

Density:

Dynamic viscosity: Pressure (variable):

\section{$G_{\nu}$}

$V_{\nu}$

$I_{\nu \mu}$

$u_{\nu}, v_{\nu}, w$

$h$

$\rho(p)$

$\eta(p)$

$p$

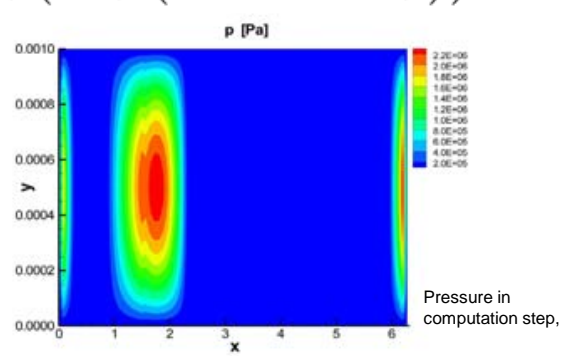

In the simulation we get cavitation for a crack with microstructured surface. We compute a test step to detect the areas of cavitation. There the pressure is set to zero in the following computation step. We choose $u_{1}=100000 \mathrm{~m} / \mathrm{s}$ which yields a Reynolds number of 24500 . We compute on a coarse grid with $81 \times 161$ nodes given by our partner, IMK Kassel. For the computation step, we get a maximum relative estimated error of $0.633 \mathrm{E}-05$ and a mean error of $0.224 \mathrm{E}-06$ for $p$.

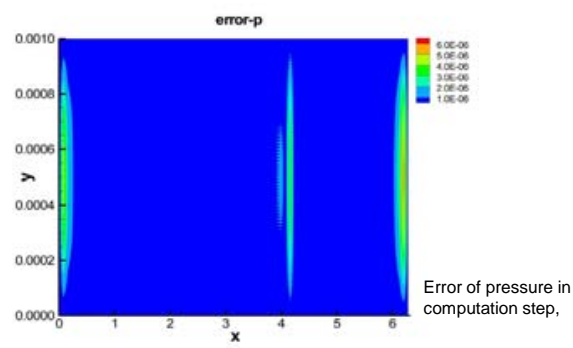

We carry out computations on grids with different microstructure of the surface. For weak scaling we get excellent scalability results for up to 1,024 processors of the SGI Altix 4700 at the Leibniz Supercomputing Centre in Garching.

For grid with 6,77 million unknowns and 4,51 million elements: 752 sec elapsed time on 1,024 processors.

This problem resulted from a cooperation of the SCC and the Institut für Maschinenelemente und Konstruktionstechnik (IMK) at the University of Kassel. 